\title{
Las redes sociales en educación: desde la innovación a la investigación educativa
}

\section{(Social networks in education: from innovation to educational research)}

\author{
Verónica Marín-Díaz \\ Universidad de Córdoba, UCO (España) \\ Julio Cabero-Almenara \\ Universidad de Sevilla, US (España) \\ Coordinadores del Monográfico
}

DOI: http://dx.doi.org/10.5944/ried.22.2.24248

\section{Cómo referenciar este artículo:}

Marín-Diaz, V., y Cabero-Almenara, J. (2019). Las redes sociales en educación: desde la innovación a la investigación educativa? RIED. Revista Iberoamericana de Educación a Distancia, 22(2), pp. 25-33. doi: http://dx.doi.org/10.5944/ ried.22.2.24248

\section{Resumen}

Las redes sociales son uno de los instrumentos básicos utilizados en la sociedad del conocimiento, y sobre todo en los más jóvenes, de manera que algunas personas empiezan a calificar a las nuevas generaciones como la "generación muda", pues sus conexiones las realizan fundamentalmente a través de texto escrito en redes sociales. Tal presencia en la sociedad ha repercutido también para un crecimiento exponencial en los entornos de formación, donde los centros las incorporan, no solo para tareas administrativas y de información a las familias, sino también los docentes como herramientas e instrumentos, para transmitir información y crear entornos de trabajo colaborativos. Tal utilización ha venido potenciada por la extensión de los dispositivos móviles entre los estudiantes y la presencia de las redes inalámbricas en los centros educativos. Ahora bien, frente a esta presencia, su incorporación a la enseñanza adolece de la problemática de contar con investigaciones que nos aporten modelos y "buenas prácticas" para su utilización. Al mismo tiempo, también encontramos hándicaps como la visión negativa que se puedan tener de ellos tanto por parte del alumno como del docente, así como la visión social que en un momento dado pueden llegar a tener fuera del ámbito académico, la nula o escasa seguridad que dan al usuario si no configura adecuadamente la privacidad, ciertos riesgos de adicción o uso problemático de las redes, o falta de control sobre lo escrito o subido. Y son algunos de los aspectos los que se analizan en el presente monográfico. 
Palabras clave: redes sociales, alumno, aprendizaje, trabajo colaborativo, adicción.

\begin{abstract}
Social networks are some of the basic tools utilized by the Knowledge society, especially by the youngest population, such that some people have begun to label the new generations as the "mute generation", as their personal connections are made mainly through the written text in social networks. Such presence in society has also resulted in the exponential growth in training environments, where centres have included it not only in administrative and information tasks and information for the family, but the teachers have also utilized them as tools and instruments for broadcasting information and for creating collaborative work environments. This use has been promoted by the spread of mobile devices among students and the presence of wireless networks in education centres. However, when dealing with this presence, their addition to education has suffered from the problem of counting with research studies that provide us with models and "Good practices" for their use. At the same time, some handicaps have also been found, such as the negative view given to them or to their use by the students as well as the teachers, as well as the social view that in a given moment they could have outside of the education sphere, the null or scarce security given to users if privacy is not configured properly, some addiction risks or problematic use of the networks, or the lack of control about what has been written or uploaded. These are some of the aspects that are analysed in this monograph.
\end{abstract}

Keywords: Social networks, student, learn, collaborative work, addiction.

Vivimos en un mundo conectado, la red de redes, Internet, forma parte de nuestra idiosincrasia, es un elemento clave en nuestro día a día. Su rápido desarrollo ha propiciado que sea reconocida de manera positiva tanto por la sociedad en general como por la comunidad académica en particular (Gialamas, Nikolopoulou y Koutromanos, 2013).

Poniendo nuestra atención en la esfera educativa, encontramos que la predisposición de los alumnos de todos los niveles educativos a conectarse a Internet, abre la puerta a que las herramientas 2.0 que bajo su arquitectura se van generando, se incorporen no solo como meros recursos en la forma en que los procesos de enseñanza y aprendizaje se van a ir construyendo, sino que se pueden llegar a convertir en protagonistas del proceso formativo.

En ese escenario, las redes sociales van cobrando una gran relevancia dado que, si las entendemos como un catalizador de las pulsiones humanas y generador de relaciones, se podría decir que realmente han supuesto más que una revolución tecnológica una de carácter social, brindando nuevas maneras a todos los internautas de ser creadores y aprendices (Holcomb y Beal, 2010), produciéndose un continuo proceso de crecimiento intelectual, social y emocional. Ya en 2012, Laru, Näykki y Järvelä indicaban que los mecanismos que presentan las herramientas 2.0 más 
allá de ser insignificantes, eran y son per sé uno de los recursos que más han transformado y transformarán el proceso de aprendizaje, de ahí la necesidad de no hacerlas desaparecer de universo educativo y social.

El crecimiento de la red de redes ha traído de la mano, que, herramientas como el software social, se hayan ido incorporando poco a poco en las dinámicas que los docentes desarrollan en sus aulas (Cabero y Marín, 2013, 2014). Así, estudios como el de Brenley y Coven (2018) y Awidi, Paynter y Vujosevic (2019), señalan que medios sociales como Facebook, Twitter y Youtube ofrecen un abanico de oportunidades para que los usuarios interactúen y compartan información, no solo con sus amigos y familia, sino también con personas con las que tienen algo en común, de ahí que su imbricación con la educación sea sentida como un complemento a la docencia y al aprendizaje (Tuñez y Sixto, 2012; Rodríguez, López y Martín, 2017; Ruíz, 2019).

Debemos ser conscientes de que inicialmente no nacieron con la intención de ser consideradas un recurso educativo (Fueyo, Braga y Fano, 2015), dado que su creación se basó en la teoría de los seis grados de proximidad de Frigyes Karinthy (1930), siendo denominada así por Watts en 2003 en su obra Six degrees: The science of a connected age; su pilar era el establecer relaciones entre los internautas, de ahí que una de las principales necesidades del ser humano, estar comunicado, se viera cubierta desde el primer momento de su aparición en el escenario de Internet.

Trabajos como el estudio realizado por Rial, Gómez, Braña y Varela (2014), donde se indicaba como el 67,5\% de los estudiantes adolescentes gallegos se conectaba a las redes sociales para hablar con sus amigos y familiares avalan la idea de adjudicarle un perfil académico. En definitiva, vemos el objetivo con el que se creó, realmente se cubre de un lado y de otro amplía su horizonte.

La presencia de elementos como Instagram, Facebook, Linkedin o WhatsApp van dibujando no solo un nuevo perfil en la forma de entender la práctica educativa, sino también en las relaciones que se establecen entre los estudiantes y de estos con sus profesores.

Por otra parte, la forma en que se plantean las metodologías a llevar a cabo durante el acto docente, cobra una visión diferente al incorporar las redes sociales, pues el conocimiento adquiere una nueva dimensión y se acerca al estudiante en cualquier momento y situación, dado que puede ser reconstruido de manera continua y favoreciendo el desarrollo de otras formas de este, además de nuevas perspectivas de aprendizaje (Wilson, 2014).

Otorgar a las redes sociales un calificativo como el de educativas, supone que deben asumir por parte de todos los actores implicados en el proceso de uso, que el punto de énfasis pivota en la colaboración, la cooperación y la comunicación. Como afirman González y Muñoz (2016, p. 85) "el eje de todas ellas se adscribe a la interacción y capacidad de responder y comunicar con rapidez y elocuencia”.

El auge del software social también presenta limitaciones en la acción educativa, algunas de ellas vinculadas a la disposición de dispositivos capaces de realizar todo aquello que se desea en el momento y lugar que queremos. Por otra parte, también 
encontramos hándicaps como la visión negativa que se puedan tener de ellos, tanto por parte del alumno como del docente, así como la perspectiva social que en un momento dado pueden llegar a tener fuera del ámbito académico, la nula o escasa seguridad que dan al usuario si estos no configuran adecuadamente la privacidad, ciertos riesgos de adicción o uso problemático de las redes, falta de control sobre lo escrito o subido, fomentar la pereza.... (Durán y Guerra, 2015; González y Muñoz, 2016; Valencia y Castaño, 2019).

No obstante, este monográfico quiere resaltar también los aspectos positivos que tiene la presencia en la academia de las redes sociales. Como señala Chávez (2015, p. 86) el uso de las redes sociales en educación conlleva que las metodologías de aula "promueven el trabajo colaborativo, incrementa la motivación y el rendimiento académico, aumenta y diversifica el conocimiento y mejora la retención de lo aprendido", en definitiva, permiten la colaboración, el intercambio de ideas, de conocimiento y el crecimiento intelectual y educativo del alumno en primera instancia y del docente en segunda.

\section{OPORTUNIDADES DE APRENDIZAJE A TRAVÉS DE LAS REDES SOCIALES}

En este monográfico se invita a presentar artículos vinculados a la utilización de las redes sociales en las aulas desde dos perspectivas, una como elemento de innovación de este acto educativo, en el que se refleje cómo su empleo puede dinamizar la metodología activa del proceso de enseñanza-aprendizaje; y por otra parte se pretende presentar resultados de investigación vinculados a la presencia y uso de las redes sociales en las aulas, de manera que se pueda tomar el pulso a su estado en las mismas y los beneficios o no que puedan aportar a la enseñanza en general y a la construcción activa del conocimiento en particular.

Abre el monográfico el artículo titulado "Percepciones de estudiantes universitarios chilenos sobre uso de redes sociales y trabajo colaborativo", firmado por Cabero, Del Prete y Arancibia. En esta línea se confirman las aportaciones que autores como Rosli, Saleh, Aris, Ahmad, Sejzi y Shamsudin (2016) y Araujo (2019), quienes sostienen, que la incorporación de las redes sociales a la educación superior, obedece a una forma de tender puentes en la continuidad de la comunicación entre el profesor y el estudiante y entre estos y los demás alumnos. Cabero, Del Prete y Arancibia, realizan un estudio con alumnado universitario chileno, quienes manifiestan emplear las redes sociales desde una perspectiva educativa y como medio para implementar el trabajo colaborativo. Entre sus resultados principales destaca la predisposición de los estudiantes encuestados para trabajar con alumnado de otras titulaciones, de manera que se puedan tender puentes entre los diversos centros educativos. Esta idea coincide con la aportada por Tess (2013), Chávez y Gutiérrez (2016) y Garay, Tejada y Castaño (2017), quienes han trabajado con estudiantes 
españoles, de ahí que la visión extrapolable del trabajo colaborativo mediante redes sociales pueda ser aplicado a la educación superior.

Continuando con la línea universidad y redes sociales se presentan las limitaciones o problemas que puede tener el empleo de estas en este nivel académico. Vilches y Reche, bajo el título "Limitaciones de WhatsApp para la realización de actividades académicas colaborativas en la Universidad", presentan las posibles dificultades que esta herramienta puede tener en la metodología docente. Es significativo que casi dos décadas después de lo que ya recogían Harasim, Hiltz, Turoff y Trieles (2000), este artículo ponga sobre el tapete que las limitaciones de hace tiempo continúan hoy, y no parece que vayan a reducirse, abriendo así, una línea de trabajo e investigación para promover una mejora en el uso de las redes en general y de WhatsApp en particular en las universidades y centros escolares. Aspectos como la mala interpretación de los mensajes y la difusión de información ajena al tema del grupo, reflejados también en el trabajo de Lafaurie-Molina, Sinning-Ordóñez y Valencia-Cobo (2018) son sus principales resultados.

Cierra esta primera propuesta de trabajo con redes sociales la que firma Rosabel Roig, bajo el título de "Repercusión de las metodologías activas del Aprendizaje basado en Proyectos (ABP), Flipped Classroom y Gamificación en Twitter". La idea central de Roig no es otra que concretar el valor de un tuit. Mediante la combinación de tres metodologías docentes centraliza el objeto de estudio en esclarecer cómo los usuarios de Twitter van posicionándose de manera relevante en el universo de la red. Al hilo de las ideas que Shane-Simpson, Manago, Gaggi, y Gillespie-Lynch (2018) sobre Twitter como red social, se presenta una nueva forma de ver y entender esta como elemento que promueva el aprendizaje basado en proyectos. Este artículo refleja los datos que Devendra (2015), mostraba en su investigación, al indicar que su influencia en la metodología de trabajo en el aula se producía de forma rápida, permitiendo la exposición general de ideas y su trasmisión, al igual que aporta Roig en su investigación.

Poniendo el acento en una segunda visión del empleo de las redes sociales, este monográfico profundiza en la visión académica que tienen en su más pura extensión, a partir del meta-análisis realizado por Jesús Salinas y Victoria Marín, y bajo la denominación "Meta-síntesis cualitativa sobre colaboración científica e identidad digital académica en redes sociales". En este los autores nos expondrán la escasez de trabajos de investigación sobre prácticas académicas con las redes sociales al igual que Mnkandla y Minnaar (2017), no obstante, el trabajo de Salinas y Marín aporta la perspectiva critica, en concreto identificaron artículos que versaban sobre los propósitos de colaboración científica y el cuidado de la identidad digital académica. Para ello han realizado una exhaustiva revisión en cinco bases de datos internacionales, de las que extrajeron 68 aportaciones publicadas desde 2008 a 2018. Se ha concluido con la necesidad de realizar investigaciones que versen sobre: networking, el intercambio de conocimiento y la identidad digital. 
Una línea importante de trabajo en el área de las redes sociales, viene de la mano de las actitudes y usos que los jóvenes hacen de ellas. En una primera aportación firmada por Tejada, Castaño y Romero se pone de relieve como los preadolescentes se mueven en las redes sociales. Con el título "Los hábitos de uso en las redes sociales de los preadolescentes", los autores ponen de manifiesto que los preadolescentes no tienen nociones para "moverse" en las redes sociales, pues no reciben una formación que les ayude a realizar un uso y una navegación por las mismas seguro, datos coincidentes con el trabajo de Prats, Torres-Rodríguez, Oberst y Carbonell (2018).

Cierra este monográfico el aspecto más contradictorio que tienen las redes sociales, que viene de la mano de los hábitos de uso de estas por parte de los estudiantes. Bajo la perspectiva de los riesgos que conlleva su empleo encontramos de un lado el estudio llevado a cabo por Marín-Díaz, Vega-Gea y Passey (Determinación del uso problemático de las redes sociales por estudiantes universitarios). Los autores tratan de esclarecer el nivel de uso problemático que los alumnos universitarios pueden llegar a presentar. Es relevante que los estudiantes participantes en este estudio han reflejado no sentir o presentar una conducta adictiva hacia las redes sociales al igual que sucedía en los trabajos de Marín, Vega y Sampedro (2018) y el de Durak y Seferoglu (2019).

De otro lado y en línea con esta apuesta, se encuentra la última aportación firmada Maldonado, García y Sampedro (El efecto de las TIC y redes sociales en estudiantes universitarios), quienes nos presentan la visión de los estudiantes mexicanos sobre el uso de las TIC y de las redes sociales en su vida académica. En referencia y en línea con el trabajo de Marín-Díaz, Vega-Gea y Passey, este artículo nos adentra de manera relevante en cómo la universidad en la que se realizan los estudios superiores va a determinar un cambio en la conducta de los estudiantes, como reflejan Ramírez, Cejas y Sánchez (2017).

\section{REFERENCIAS}

Araujo, J. C. (2019). El componente social. Un indicador del trabajo colaborativo online. EDMETIC, Revista de Educación Mediática y TIC, 8(1), 171-200. doi: https://doi.org/10.21071/edmetic. v8i1.11104

Awidi,I.T.,Paynter,M.,yVujosevic, T.(2019). Facebook group in the learning design of a higher education course: An analysis of factors influencing positive learning experience for students. Computers \& Education, 129, 106-121, doi: https://doi. org/10.1016/j.compedu.2018.10.018

Brenley, D. B., y Covey, J. (2018). Risky behavior via social media: the role of reasoned and social reactive pathways. Computers in Human Behaviour, 78, 183-191. doi: https://doi.org/10.1016/j. chb.2017.09.036

Cabero, J., y Marín, V. (2013). Latin American university students' perceptions of social networks and group work. RUSC. Universities and Knowledge Society Journal, 1O(2), 462-477. Recuperado de http://journals.uoc.edu/ojs/index.php/ rusc/article/view/v1on2-cabero-marin

Cabero, J., y Marín, V. (2014). Posibilidades educativas de las redes sociales y el trabajo en grupo. Percepciones de los alumnos universitarios. Comunicar, 42, 
165-172. doi: http://dx.doi.org/10.3916/ C42-2014-16

Chávez, I., y Gutiérrez, M. C. (2015/16). Redes sociales como facilitadoras del aprendizaje de ciencias exactas en la educación superior. Apertura, 7(2), 1-112. Recuperado de http://www.udgvirtual. udg.mx/apertura/index.php/apertura/ article/view/698/499

Chávez, J. J. (2015). Uso de las redes educativas en la educación superior. Un caso específico. ComHumnitas: Revista Científica de Comunicación, 6(1), 82-96. Recuperado de http:// www.comhumanitas.org/index.php/ comhumanitas/article/view/20158

Devendra, M. (2015). Impact of social media on teaching and teaching of visual communication-A contemporary Analysis. International Journal of Science, Technology and Humanities, 2, 86-90.

Durak, H. Y., y Seferoglu, S. (2019). Modeling of variables related to problematic social media usage: Social desirability tendency example. Scandinavian Journal of Psychology, o5 March. doi: 10.1111/ sjop.12530.

Durán, M., y Guerra, J. M. (2015). Uso y tendencias adictivas de una muestra de estudiantes universitarios españoles a la red social: la actitud positiva hacia la presencia de la madre en la red como factor protector. Anales de Psicología 31(1), 260-267. doi: https://doi.org/10.6018/ analesps.31.1.158301

Fueyo, A., Braga, G., y Fano, S. (2015). Redes sociales y educación: el análisis socio-político como asignatura pendiente. Revista Interuniversitaria de Formación del Profesorado, 82(29.1), 119-129. Recuperado de http://aufop.com/aufop/ uploaded files/articulos/1507479473.pdf

Garay, U., Tejada, E., y Castaño, C. (2017). Percepciones del alumnado hacia el aprendizaje mediante objetos educativos enriquecidos con realidad aumentada.
EDMETIC, Revista de Educación Mediática y TIC, 6(1), 145-164. doi: https://doi.org/10.21071/edmetic. v6i1.5812

Gialamas, V., Nikolopouiou, K., y Kutromanos, G. (2013). Student teachers' perceptions about the impact of internet usage on their learning and jobs. Computers \& Education, 62, 1-7. doi: http://dx.doi.org/10.1016/j. compedu.2012.10.012

González, C., y Muñoz, L. (2016). Redes Sociales su impacto en la Educación Superior: Caso de estudio Universidad Tecnológica de Panamá. Campus Virtuales, 5(1), 84-90. Recuperado de http://uajournals.com/ojs/index.php/ campusvirtuales/article/view/117/106

Harasim, L., Hiltz, S.R., Turoff, M., y Trieles, L. (2000). Redes de aprendizaje. Guía para la enseñanza y el aprendizaje en red. Barcelona: Gedisa.

Holcomb, L. B. \& Beal, C. M. (2010). Capitalizing on web 2.0 in the social studies context. TechTrends, 54 (4), 2832. doi: https://dx.doi.org/10.1007/ s11528-010-0417-0

Lafaurie-Molina, A. M., Sinning-Ordoñez, P. A., y Valencia-Cobo, J. A. (2018). WhatsApp y Facebook como mediación pedagógica en procesos de Orientación Socio Ocupacional. Educación y Educadores, 21(2), 179-199. doi: https:// dx.doi.org/10.5294/edu.2018.21.2.1

Laru, J., Näykki, P., y Järvelä, S. (2012). Supporting small-group learning using multiple web 2.0 tools: a case study in the higher education context. Internet and Higher Education 15, 29-38. doi: 10.1016/ jhedu.2011.08.004.

Mnkandla, E., y Minnaar, A. (2017). The Use of Social Media in E-Learning: A Metasynthesis. The International Review of Research in Open and Distributed Learning, 18(5), 227-248. doi: https:// doi.org/10.19173/irrodl.v18i5.3014 
Prats, M. A., Torres-Rodríguez, A., Oberst, U., y Carbonell, J. (2018). Diseño y aplicación de talleres educativos para el uso saludable de internet y redes sociales en la adolescencia: descripción de un estudio piloto. Pixel Bit, Revista de Medios y Educación, 52, 111-124. doi: https:// dx.doi.org/10.12795/pixelbit.2018.i52.08

Ramírez, L. M., Cejas, S., y Sánchez, M. (2017). Las redes sociales potencializan $\mathrm{u}$ obstaculizan el desarrollo educativo de los jóvenes en la Ciudad de Puebla. $X I$ Congreso de la Red Internacional de Investigadores en Competitividad, 11, 1106-1118. Recuperado de https:// riico.net/index.php/riico/article/ download/1493/1153

Rial, A., Gómez, P., Braña, T., y Varela, J. (2014). Actitudes, percepciones y uso de Internet y las redes sociales entre los adolescentes de la comunidad gallega (España). Anales de Psicología, 3o(2), 642-655. doi: https://doi.org/10.6018/ analesps.30.2.159111

Rodríguez, M., López. A., y Martín, I. (2017). Percepciones de los estudiantes de ciencias de la educación sobre las redes sociales como metodología didáctica Pixel Bit, Revista de Medios y Educación, 50, 77-93. doi: http://dx.doi.org/10.12795/ pixelbit.2016.i50.05

Rosli, M., Saleh, N. S., Aris, B., Ahmad, M., Sejzi, A., y Shamsudin, N. (2016). E-Learning and Social Media Motivation Factor Model. International Education studies, 9(1), 20-30. doi: 10.5539/ies. v9n1p2o.

Ruiz, A. (2019). Competencia digital y TICs en interpretación: «renovarse o morir». EDMETIC, Revista de Educación Mediática y TIC, 8(1), 55-71. doi: https:// doi.org/10.21071/edmetic.v8i1.11062

Shane-Simpson, C., Manago, A., Gaggi, N., y Gillespie-Lynch, K. (2018). Why do college students prefer Facebook, Twitter, or Instagram? Site affordances, tensions between privacy and self-expression, and implications for social capital. Computer in Human Behaviour, 86, 276-288. doi: https://doi.org/10.1016/j. chb.2018.04.041

Tess, P. A. (2013). The role of social media in higher education classes (real and virtual) - A literature review. Computers in Human Behaviour, 29, A6o-A68. doi: https://doi.org/10.1016/j. chb.2012.12.032

Tuñez, M., y Sixto, J. (2012). Las redes sociales como entorno docente: análisis del uso de Facebook en la docencia universitaria. Pixel Bit, Revista de Medios y Educación, 41, 77-92.

Valencia, R., y Castaño, C. (2019). Use and abuse of social media by adolescents: a study in México. Pixel Bit, Revista de Medios y Educación, 54, 7-28. doi: https://doi.org/10.12795/pixelbit.2019. i54.01

Watts, D. J. (2003). Six degrees: The science of a connected age. New York city: Editorial W. W. Norton \& Company.

Wilson, K. B. (2014). Impact of emerging technologies on teacher education: experiences of teacher trainees. Journal of Education and Practice, 5(28), 168176.

\section{PERFIL ACADÉMICO Y PROFESIONAL DE LOS AUTORES}

Verónica Marín-Díaz. Profesora Titular de Universidad de la Facultad de Ciencias de la Educación de la Universidad de Córdoba, ha sido Directora del Máster en Educación Inclusiva de la citada universidad en el período 2012-2015. Directora del grupo PAI SEJ-623-EDMETIC, Grupo de investigación en Educación Mediática 
y TIC., con sede en la Universidad de Córdoba (España) Miembro del grupo de investigación de Tecnología Educativa de la Universidad de Sevilla. Editora de la revista internacional EDMETIC, Revista de Educación Mediática y TIC (http:// www.uco.es/ucopress/ojs/index.php/edmetic/index). Sus líneas de investigación giran en torno a las tecnologías emergentes.

E-mail: vmarin@uco.es

Dirección:

Facultad de Ciencias de la Educación

Universidad de Córdoba

Facultad de Ciencias de la Educación

Avda. San Alberto Magno s/n 14004

Córdoba (España)

Julio Cabero-Almenara. Catedrático de Tecnología Educativa de la Universidad de Sevilla. Premio de la Real Maestranza de Sevilla (España). Director del grupo PAI GID: Grupo de Investigación Didáctica. Director de la revista Pixel Bit, Revista de Medios y Educación (https://recyt.fecyt.es/index.php/pixel/index). Director del grupo de investigación de Tecnología Educativa de la Universidad de Sevilla. Sus líneas de investigación giran en torno a las tecnologías emergentes. E-mail: cabero@us.es

Dirección:

Faculta de ciencias de la Educación

Universidad de Sevilla

Facultad de Ciencias de la Educación

Calle Pirotecnia s/n 41013

Sevilla (España)

Fecha de recepción del artículo: 16/03/2019

Fecha de aceptación del artículo: 18/04/2019

Fecha de aprobación para maquetación: 26/04/2019 